\title{
How Often Should We Believe Positive Results? Assessing the Credibility of Research Findings in Development Economics
}

\author{
Aidan Coville* \\ The World Bank \\ Eva Vivalt ${ }^{\dagger}$ \\ The Australian National University
}

November 1, 2017

\begin{abstract}
Under-powered studies combined with low prior beliefs about intervention effects increase the chances that a positive result is overstated. We collect prior beliefs about intervention impacts from 125 experts to estimate the false positive and false negative report probabilities (FPRP and FNRP) as well as Type S (sign) and Type $\mathrm{M}$ (magnitude) errors for studies in development economics. We find that the large majority of studies in our sample are generally credible. We discuss how more systematic collection and use of prior expectations could help strengthen the literature.
\end{abstract}

\footnotetext{
*E-mail: acoville@worldbank.org.

†E-mail: eva.vivalt@anu.edu.au.
} 


\section{Introduction}

A replication of 18 laboratory experiments previously published in the American Economic Review and Quarterly Journal of Economics found that $61 \%$ of the results supported their original findings (Camerer et al., 2016). A similar exercise in psychology found less than half of the original results from 100 studies fell within the $95 \%$ confidence interval of the replication study (Open Science Collaboration, 2015). There are three broad reasons for why research findings may not replicate. First, there may be changes in implementation or context between the original study and the replication. Second, biases can enter into the research process. For example, specification searching or publication bias can lead to an over-representation of positive effects (Brodeur et al., 2016; Vivalt, 2017a), while the "file-drawer problem" means that non-significant results often fail to see the light of day (Rosenthal, 1979). The third reason for low reproducibility - and the focus of this paper is statistical in nature.

While it is well known that an under-powered study reduces the likelihood of identifying an effect when it is present (Type I error), it is less appreciated that low power also increases the chance that a result is spurious and exaggerated when a significant impact is found (Gelman \& Carlin, 2014). Two studies with identical, statistically significant pvalues could have substantially different false positive report probabilities, depending on the power of the study. Despite the academic norm of considering $80 \%$ to be a signal of an appropriately-powered study, reviews for the medical, economic and general social sciences literature estimate median power to be in the range of $8 \%-24 \%$ (Button et al. 2013; Ioannidis et al., forthcoming; Smaldino \& McElreath, 2016). This has the potential to substantially influence the reliability and interpretation of significant effects. To estimate the likelihood that a statistically significant effect is spurious, Wacholder et al. (2004) introduce the false positive report probability (FPRP) which is an application of Bayes' rule that makes use of a study's power, the significance level, and the prior belief that an intervention is likely to have a meaningful impact. Ioannidis (2005) draws on this framework to 
estimate that more than half of the significant published literature in biomedical sciences could be false, and a recent paper by Ioannidis et al. finds $90 \%$ of the economic literature is under-powered (forthcoming). The current literature on this topic has primarily focused on psychology and medicine (e.g. Begley \& Ioannidis, 2015) and has relied on existing data as inputs into the false positive estimations. We contribute to this literature by collecting priors data from topic experts to explore the prevalence of false positive reporting in development economics. We also explore what Gelman \& Tuerlinckx (2000) have termed errors of sign (Type S errors) and magnitude (Type M errors), respectively the probability that a given significant result has the wrong sign and the degree to which it is likely exaggerated.

We gather up to five predictions from each of 125 experts covering 130 different results across typical interventions in development economics and generate estimates of the likelihood of false reporting and exaggeration of the effects of significant results. We complement this with an exercise that collects predictions of main results from 4 impact evaluations presented at the World Bank between February and June 2017 as part of the Development Impact Evaluation (DIME) unit's lunchtime seminar series and similarly estimate power and false reporting likelihoods.

There are at least three important differences in development economics when compared to psychology, medicine, or even other fields within economics. First, while there is great heterogeneity in costs across studies, the marginal cost of increasing sample size can often be low. For example, the GiveDirectly unconditional cash transfer program in Kenya can afford to target all individuals in 300 villages, a scale that would be prohibitively expensive in the United States. While not all studies may have low marginal costs, studies in development economics often have larger sample sizes than clinical trials or other economics studies and may consequently be somewhat better-powered on average. Second, it is likely that across-study variation, which is heavily influenced by program implementation in the field and where there is typically substantial heterogeneity in intervention quality, is larger than for more controlled laboratory experiments. This suggests that we will have to be cautious about pooling results on a given intervention-outcome combination; for example, 
despite being more susceptible to bias, in our context random-effects meta-analyses may be more appropriate than fixed-effect meta-analyses. Third, there are far fewer studies in development economics than in psychology or medicine. Thus, if we want to leverage a set of results on the same intervention-outcome combination, we are forced to limit our attention to those intervention-outcome combinations where there is a critical mass of studies to review.

Our findings in this subset of studies are less dramatic than estimates for other disciplines. Median power is estimated to be between $18 \%$ and $59 \%$. Experts predict that interventions will have a meaningful impact approximately $60 \%$ of the time. We calculate the median FPRP to be between 0.001 and 0.008, compared to the median significant p-value of 0.002 . The average value for the false negative report probability (FNRP) is 0.512-0.624 depending on the specification used. The likelihood of a significant effect having the wrong sign (Type S error) is close to 0 while the median exaggeration factor (Type M error) of significant results is estimated to be between 1.2 and 2.2.

The remainder of the paper proceeds as follows: Section 2 describes the current approaches to identifying false reporting, Section 3 presents the framework for our analysis, Section 4 describes the data, and Section 5 presents results. We discuss the implications of the results in Section 6 and conclude in Section 7.

\section{Estimating the extent of false reporting}

Wacholder et al. (2004) provide formulas for the FPRP and FNRP that are derived from a simple application of Bayes' rule and require estimates of statistical power, the significance level of a result, and the prior assigned to the probability that an intervention is effective. This approach determines the likelihood that an observed statistically significant effect represents a true underlying effect and requires one to set a specific threshold for an effect to be "true". The FPRP can be shown to be equal to:

$$
F P R P=p(1-\pi) /[p(1-\pi)+(1-\beta) \pi]
$$


where $p$ is the p-value obtained in the study, $\beta$ is the Type II error rate, and $\pi$ is the prior probability of a non-null effect for the given threshold (Wacholder et al., 2004). The FNRP analogously is:

$$
F N R P=\beta \pi /[\beta \pi+(1-p)(1-\pi)]
$$

Related work by Gelman \& Carlin (2014) argues that we may expect most interventions to have some type of an effect, but that these effects could be very small, in which case the presentation of a dichotomous inference, rejecting or accepting a null hypothesis, may be misleading. From this perspective, the problem is not about identifying an effect when one does not exist, but rather incorrectly estimating the effect. This can create two estimation errors when interpreting significant results: a significant effect having the wrong sign (Type S error) or magnitude (Type M error). Their approach requires the researcher to (i) provide a best estimate of the true underlying effect, $\hat{\theta}$ and (ii) define a random variable $d^{r e p}$ that represents a hypothetical study replication under the exact same conditions. In this case, $d^{r e p}$ would have the same standard error as the original study, but be centered on a mean of $\hat{\theta}$. It is then possible to estimate the probability of the hypothetical replication finding a significant effect (i.e. being larger in absolute value than the critical significance level in the original hypothesis test). A Type $\mathrm{S}$ error can be calculated as the probability of the replication finding a significant effect in the opposite direction to the hypothesized true value divided by the probability of finding a significant effect. Finally, the Type M error can be interpreted as an exaggeration factor which is the expected value of the replication effect if it is significant divided by the hypothesized true effect.

Estimating these errors raises the spectre of ex post power calculations. Hoenig \& Heisey (2001) articulate the dangers of ex post power calculations that use study data as inputs for the parameters needed for the estimate and the logical inconsistencies that it can create. In particular, they note that the observed p-value is a 1:1 function of observed power, and thus attempting to estimate power using the observed estimate as the threshold and the observed p-value as $\alpha$ will yield a deterministic value for power and thus fail to contribute 
beyond what is already captured from the p-value. Gelman \& Carlin (2014) acknowledge this and, by using external information on the hypothesized intervention impact, are able to circumvent this concern.

In particular, to calculate the power, FPRP, FNRP, Type S and Type M errors of a study, some level of information that is external to the study data itself is required, namely, an informed expectation of the intervention's "true" impact. Calculating the FPRP and FNRP relies on an informed estimate of the likelihood that the true impact lies above a certain meaningful threshold, while calculating Type $\mathrm{S}$ and Type $\mathrm{M}$ errors requires an estimate of what the true effect is (regardless of whether it is meaningful).

There are three common approaches that have been used to estimate potential effect sizes ex ante for the purpose of calculating power, FPRP, FNRP, Type S and Type M errors. In the first case, authors use the currently available evidence and theory on the topic to identify informed parameter ranges. This approach has been used for both FPRP and FNRP calculations (Wacholder et al. 2004; Ioannidis, 2005) as well as Type S and M error estimates (Gelman \& Carlin, 2014). While helpful in providing back-of-the-envelope estimates, it relies on good parameter estimates and may be more useful as a bounding exercise given the parameter ranges. The second approach uses meta-analysis results, when they exist, as best estimates of the underlying intervention impact. These are then used to estimate study power. For instance, Button et al. (2013) estimate a median power of $21 \%$ in neuroscience while Ioannidis et al. (forthcoming) find the median power in various economics publications to fall within a range of $8-18 \%$. It is possible, however, that using meta-analysis results as benchmarks may overestimate the true effect, particularly in the presence of publication bias. This approach may also be challenging for the development economics setting given the relatively few topics with enough results to generate credible meta-analyses and the inherent heterogeneity of development projects. The third approach involves eliciting priors on intervention effects directly from experts in the topic. Here, we can draw on lessons from the relatively new literature on this topic, which has mostly focused on gathering priors for the sake of estimating predictability of results rather than 
using them as an input for estimating research credibility. The first stylized fact in this literature is that individuals systematically overestimate program impacts. This is true of academics (Groh et al., 2016; DellaVigna \& Pope, 2016a), government policymakers (De Andrade et al., 2014), private sector implementers (Hirshleifer et al., 2015), and actual program participants (McKenzie, forthcoming). The second key finding in this literature is that there is wisdom in crowds. DellaVigna \& Pope (2016b) design an experiment that tests 18 different economic and behavioral incentives to motivate effort and then elicits priors regarding intervention effects from experts and lay respondents. Aggregated responses provide better predictions than $96 \%$ of individual responses, while the level of expertise and familiarity with the subject matter has an ambiguous relationship with accuracy and depends on the way accuracy is measured. An alternative and promising approach to elicit priors is to create a prediction market where experts are able to bet on the likely outcomes of a future study. Dreber et al. (2015) elicit estimates from experts on the likelihood that a particular result will hold in a replication exercise of psychology studies. They compare the accuracy of survey predictions to the results of a market where individuals could bet

on whether a result would be replicated. While the authors find the market yields more accurate predictions than survey responses, a similar framework was used by Camerer et al. (2016) for economics replications and found no difference between the approaches.

In this paper we use both meta-analysis results and aggregated priors to estimate power, for robustness.

\section{Method}

\subsection{Study selection and representativeness}

We leverage AidGrade's database of impact evaluation results, gathered in the course of meta-analyses of 20 different types of interventions in development economics, covering 635 studies. The reader is referred to Vivalt (2017b) for a more complete description of these data. Notably, the results in this database are categorized by type of intervention (e.g. 
conditional cash transfers) and by outcome (e.g. school enrollment rates). We start with a list of all intervention-outcome combinations covered by at least 5 papers. ${ }^{1}$ For those interventions with multiple outcomes covered by at least 5 papers, we use the outcome that was covered by the greatest number of studies. This yields the list provided in Appendix Table A1. The process for selection of interventions for initial inclusion in AidGrade's database was arguably unbiased: from a large list of potential topics, those with the greatest number of papers on the topic were selected as finalists, and these were then quasi-randomized into the selected set. ${ }^{2}$

\subsection{Expert predictions}

We surveyed researchers to elicit their prior expectations of intervention effects and the minimum effects they would require a study to find in order for its results to be considered meaningful.

Researchers were identified as any person that had co-authored an impact evaluation for a specific program, using AidGrade's database of impact evaluations. We reached out by e-mail with a link to an online survey. The survey provided respondents with a list of the studies on a particular topic that were captured in the AidGrade database and respondents were asked to indicate those with which they were already familiar. When the initial list of studies was very large, this list was broken down into randomly-selected sets. Researchers were then shown the details of up to five of the studies with which they were not familiar (either five or the number of studies with which they did not express familiarity, if this number was less than five).

The study details that were provided included background information about the context and target population, a description of the intervention, the implementer, the outcome of interest, and the baseline or endline values of the outcome variable, where available. An

\footnotetext{
${ }^{1}$ Micronutrients programs are excluded as a type of intervention as its papers are all from the health literature and including it would overwhelm the number of development economics papers.

${ }^{2}$ One topic, women's empowerment programs, was selected by public vote as described in Vivalt (2017b). A minor deviation to the protocol described led to the inclusion of 1 additional recent study on financial literacy that was not part of the original AidGrade database. However, the study received too few predictions to be included in the analysis and thus is not discussed further.
} 
Table 1: Sample Study Description

\begin{tabular}{|c|c|}
\hline Program & CESSP Scholarship Program \\
\hline Title & $\begin{array}{l}\text { Own and Sibling Effects of Conditional Cash Transfer } \\
\text { Programs: Theory and Evidence from Cambodia }\end{array}$ \\
\hline Location & Cambodia, rural \\
\hline Sample & Transfer to poor households in treated areas \\
\hline Intervention & $\begin{array}{l}\text { Equivalent monthly USD cash transfers: From } \\
\text { US } \$ 11.25 \text { per child. } \\
\text { Intervention start date: } 2005 \\
\text { Date outcome variable collected: } 2006 \\
\text { Households receive very modest transfers, equivalent } \\
\text { to between } 2 \text { and } 3 \text { percent of the total expenditures of } \\
\text { the average recipient household, conditional on school } \\
\text { enrollment for children of middle-school age. }\end{array}$ \\
\hline Implementer & Government \\
\hline Outcome & $\begin{array}{l}\text { 1. Boys aged } 7-18-\text { School enrollment } \\
\text { 2. Girls aged } 7-18-\text { School enrollment }\end{array}$ \\
\hline Baseline enrollment level & $\begin{array}{l}62.8 \% \text { (Boys) } \\
54.4 \% \text { (Girls) }\end{array}$ \\
\hline
\end{tabular}

example is provided in Table 1 for the case of the effect of a conditional cash transfer program on school enrollment rates.

\subsection{Defining parameters}

We use Equation 1 to calculate the FPRP, which requires estimates of $p, \beta$ and $\pi$. Here, we take $p$ to be the $\mathrm{p}$-value reported in the paper, or calculate it by transforming the reported standard error or confidence interval when the p-value is not reported.

The literature typically defines $\pi$ as the prior probability of a true association of a certain magnitude between two variables. As per Wacholder et al.: "The prior probability of a hypothesis can simply be the subjective answer to the question 'What is the probability of a meaningful association between a genetic variant (for analysis of a SNP) or gene (for a haplotype analysis) and a disease?'" (2004).

Let the true effect of a particular intervention on a particular outcome in a given context 
be denoted by $\theta_{i}$. The subscript $i$ can be interpreted as indexing the study or, where results for multiple subgroups were reported within the same paper, the subgroup. We collect $n$ independent expert predictions $\widehat{\theta}_{i 1}, \widehat{\theta}_{i 2}, \ldots, \widehat{\theta}_{i n}$ for each study result, where $\hat{\theta}_{i j}$ has a probability distribution function, $f_{i}\left(\hat{\theta}_{i j}\right)$, and cumulative distribution function, $F_{i}\left(\hat{\theta}_{i j}\right)$. We also collect a set of minimum detectable effect (MDEs) estimates from $n$ experts, $\delta_{i 1}, \delta_{i 2}, \ldots, \delta_{i n}$ and define $\delta_{i}$, the median MDE for a given $i$, as the minimum meaningful effect. Then the prior likelihood of a result being successful will be:

$$
\pi_{i}=1-F_{i}\left(\delta_{i}\right)
$$

While it is possible to estimate $\pi_{i}$ without parametric assumptions about how $\hat{\theta}_{i j}$ is distributed, our preferred approach assumes $\hat{\theta}_{i j}$ is normally distributed, so that $F_{i}\left(\hat{\theta}_{i j}\right)$ is the $\mathrm{CDF}$ of a normal distribution whose mean and variance we estimate using a maximum likelihood approach. Non-parametric results, which simply use the share of the distribution of expert predictions above $\delta_{i}$ as $\pi_{i}$, are included in the appendix and do not differ substantively.

Following Gelman \& Carlin (2014), we estimate power for each study result by setting the threshold effect equal to the anticipated effect of the intervention. This could come from expert responses (by study) or from meta-analysis results for each intervention-outcome combination. In the former case, we use the mean expert prediction as the threshold, $\hat{\theta}_{i}$. In the latter, we set the threshold to be the fixed-effect or random-effects meta-analysis result, $\widehat{\mu}$, which varies only by intervention-outcome. The study power to detect an impact at least the size of $\hat{\theta}_{i}{ }^{3}$ can be calculated as:

$$
1-\beta_{i}=\Phi\left(\frac{\hat{\theta}_{i}}{s e_{i}}-z_{\alpha / 2}\right)
$$

where $1-\beta_{i}$ is the power of the study result of interest, $s e_{i}$ is the standard error of the study result estimate, and $z$ is the z-statistic for a particular type 1 error rate $\alpha$. We use

\footnotetext{
${ }^{3}$ We replace $\hat{\theta}_{i}$ with $(\hat{\mu})$ when using meta-analysis means.
} 
the standard significance level of $\alpha=0.05$.

We only include responses in our analysis if we received at least 3 independent predictions for a given study result. We estimate the FPRP when the reported p-value is less than 0.05 and estimate the FNRP otherwise.

To calculate Type $\mathrm{S}$ and Type $\mathrm{M}$ errors, the only inputs we require are the estimated true effects for each study result and the standard error associated with the point estimate for each study result. As in the FPRP calculation, we estimate the true effect alternatively from the expert estimates or from the associated meta-analysis result for the particular intervention-outcome combination. We obtain standard errors directly from the papers being analyzed. These data are then used as inputs into Gelman \& Carlin's (2014) statistical procedure retrodesign() to estimate Type $\mathrm{S}$ and Type $\mathrm{M}$ errors.

Figure 1 summarizes the approaches used.

Figure 1: Method Summary

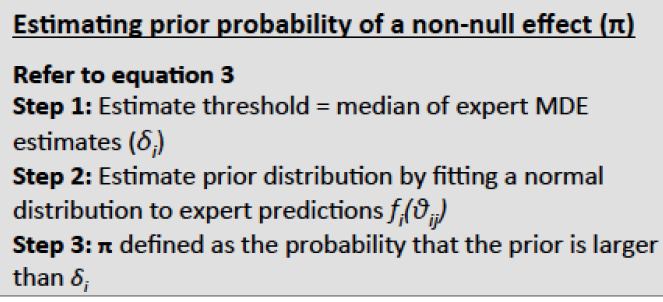

\section{Estimating power (1- $\beta$ )}

Refer to equation 4

Step 1: Set $\alpha$ to 0.05 and obtain $s_{i}$ estimates directly from study results.

Step 2: Estimate the true program effect size using either the average expert prediction for a particular study result $\left(\vartheta_{i}\right)$ or the meta-analysis mean for the intervention-outcome combination $(\mu)$.

\section{True effect estimated using:}

$\pi$ estimated using:

Method 1 (EE): expert estimates

Method 2 (FE): Fixedeffect meta-analysis

Method 3 (RE):

DerSimonian and Laird random-effects meta-

Subscript $i$ refers to a specific study result, subscript $j$ denotes each expert respondent that provides a prediction. The FPRP and FNRP are calculated using one of the three approaches described (EE, FE, $\mathrm{RE}$ ). Type $\mathrm{S}$ and $\mathrm{M}$ errors are similarly calculated using EE, FE or RE as the best estimates of the true program effect size. In the Appendix, we also present a set of results taking the raw distribution of expert predictions as the prior distribution for calculating $\pi$. 


\subsubsection{Selection of results for meta-analysis}

We must make a decision about which studies to include in the meta-analyses: all studies or only those for which we garnered a sufficient number of predictions. An argument could be made that it would be best to conduct meta-analyses based on all the studies in AidGrade's data set on a particular intervention-outcome combination, as this would fully leverage the literature. If respondents are more likely to be aware of some studies than others, then restricting attention to those studies with which they were not familiar and thus for which they were able to provide predictions could lead to selection bias. On the other hand, if there is true heterogeneity in the impact of certain programs by context, restricting attention to the set of contexts for which respondents provided predictions could lead to more accurate estimates of how a future replication would perform in those contexts. In this version of the paper, we use all studies in AidGrade's data set to conduct the fixed-effect and DerSimonian and Laird random-effects meta-analyses, as we would like to leverage the larger literature.

\subsection{Alternative approaches to prior elicitation}

DIME holds regular lunchtime seminars to present the results of new impact evaluations in development economics to a World Bank audience in Washington, D.C. We follow a similar process to calculate power and reporting error probabilities for the results covered by DIME seminars at the World Bank as we did in eliciting expert predictions, with 3 key differences: (i) none of the studies presented had been published in any form at the time of the seminar, making it very unlikely that respondents had been exposed to the results directly or indirectly; (ii) the sample comes from a wide variety of people interested in development, but not necessarily experts on the topic; and (iii) respondents were randomized into providing an estimate of impact or a minimum detectable effect, but not both, increasing the independence of these two estimates.

The advantage of this approach is that it arguably captures cleaner priors than the survey of researchers - since results had never been published, respondents were very unlikely to be even subconsciously aware of them. On the other hand, this approach covers a limited 
set of papers and the respondents are not necessarily topic experts.

Prior to each presentation, an invitation is typically sent out with a biography of the speaker and an abstract for the paper. From February to June 2017 we reviewed each DIME seminar and included them in our study if they were randomized control trials with at least one clearly defined outcome and intervention. Presentations were excluded because they were exploring general phenomena descriptively (e.g. how organizational autonomy is related to effectiveness of foreign aid implementation) or had outcomes that would not be easily assessed by respondents in the given setting (e.g. standard deviation changes in test scores). We then coordinated with the DIME media team to have invitations for the selected studies sent out without results. Instead, there was a link to a questionnaire which included a brief description of the intervention and asked readers to indicate what they thought the impact for a particular indicator was likely to be. The study description and outcome of interest was developed in conjunction with the study authors with the aim of selecting the primary outcome of interest when multiple outcomes were planned to be presented. Readers were randomly assigned to either provide an estimate of the program impact on an outcome variable or a minimum detectable effect.

\subsection{Research design transparency}

A pre-analysis plan was originally uploaded to the Open Science Framework on 10 December 2015 and last updated on 30 December 2016. Data and statistical coding files will be made available together with the pre-analysis plan at https://osf.io/5ahye. ${ }^{4}$ Data collection began in December 2016 and continued until June 2017. Analysis was conducted using Stata 14 and R 3.3.3. Departures from the pre-analysis plan are presented in an annex.

\footnotetext{
${ }^{4}$ Data and coding will be made available in a later version of this paper.
} 
Table 2: Response Rate by Intervention

\begin{tabular}{cccc}
\hline Intervention & Respondents & Contacted & Response Rate \\
\hline Bed Nets & 17 & 156 & 0.11 \\
CCTs / UCTs & 54 & 172 & 0.31 \\
Deworming & 20 & 94 & 0.21 \\
Financial Literacy & 10 & 44 & 0.23 \\
Microfinance & 16 & 75 & 0.21 \\
Water Treatment & 8 & 75 & 0.11 \\
\hline Total & 125 & 616 & 0.20 \\
\hline
\end{tabular}

\section{Data}

A total of 616 researchers were contacted for the expert predictions survey, of whom 125 responded and provided responses on up to 5 studies each. For each study, up to 3 estimates of the program impact and MDE were gathered, depending on whether the papers presented results for multiple subgroups or treatment arms. Response rates are described by intervention in Table 2. For CCTs and UCTs, we contacted those familiar with either and referred them to the same survey, which randomly asked about CCT or UCT programs, due to the substantial overlap between the topics.

Response rates varied from $11 \%$ to $31 \%$. UCTs and CCTs had both the largest pool of researchers contacted (172) and the highest response rate. As a result, $43 \%$ of respondents (54) were linked to these topics. In general, topics with a stronger health focus (bed nets, deworming and water treatment) had lower response rates than the more traditional economics topics (CCTS/UCTs, financial literacy and microfinance).

In total, we received 361 predictions covering 130 different study results across 94 papers. For 63 of these results (covering 48 papers), we obtained at least 3 estimates and this represents the full sample that we use in this paper. Of these 63, 39 had associated p-values less than or equal to 0.05 and were used for the FPRP, Type S and Type M error estimates, while the remaining 24 had p-values greater than 0.05 and were used for the FNRP calculation. Table 3 provides an intervention-level breakdown of the number of eligible study results and the number that received 3 or more predictions and were actually 
Table 3: Number of Treatments by Intervention

\begin{tabular}{ccc}
\hline Intervention & Number of Study Results & Study Results Analyzed \\
\hline Bed Nets & 10 & 7 \\
CCTs & 64 & 40 \\
UCTs & 7 & 6 \\
Deworming & 23 & 4 \\
Financial Literacy & 9 & 1 \\
Microfinance & 5 & 5 \\
Water Treatment & 12 & 0 \\
\hline Total & 130 & 63 \\
\hline
\end{tabular}

Study results are included in the analysis if we received 3 or more predictions of their study impact.

analyzed. The proportion of results analyzed varies significantly across topic, ranging from $0 \%$ (water treatment) to $100 \%$ (microfinance). CCTs and UCTs make up more than two thirds of the study results analyzed.

The DIME seminar series predictions included 8 results from 4 studies that covered the following topics: retaining maternal health providers through monetary and non-monetary incentives; using mass media campaigns (radio and print) to increase demand for quality solar lanterns; providing household connection subsidies to join the electricity grid; and providing a female-only car in metros with the aim of reducing physical and verbal harassment for females using public transport. A total of 96 program impact estimates and $67 \mathrm{MDE}$ estimates were captured.

\section{Results}

\subsection{Study selection by experts}

Figure 2 illustrates the degree of selection from the initial sampling frame to the final sample analyzed. The representativeness of our results for the broader development economics literature is influenced by three factors: (i) the representativeness of the AidGrade sampling frame; (ii) the selection criteria for study eligibility; and (iii) the expert selection process. 
Figure 2: Sample selection process

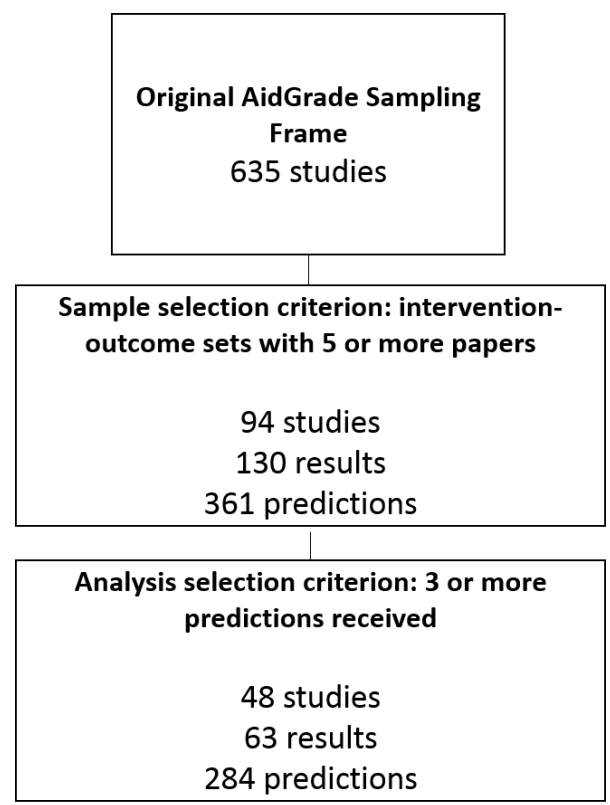

This figure describes the selection stages from the sampling frame to the final sample used for analysis.

AidGrade's sampling frame and study eligibility criteria are described earlier and we argue that our initial sample of papers is broadly representative of development economics interventions that have 5 or more impact evaluation results. However, the study selection process by experts from our sample is likely to introduce important biases. Expert respondents were presented with a list of studies on the topic and asked to indicate those studies with which they were familiar. Figure 3 shows the percent of studies from the list with which researchers were familiar.

On average, researchers had heard of $35 \%$ of studies; the median was $30 \%$. This is less than we anticipated. We are not able to distinguish between whether researchers were truly not familiar with the other studies listed or if they were unable to identify the papers based on the information provided (type of intervention, author, publication year, title of paper, program name, and country). An example of the question they received about the set of possible studies is illustrated in Figure 4. 
Figure 3: What proportion of studies were researchers familiar with?

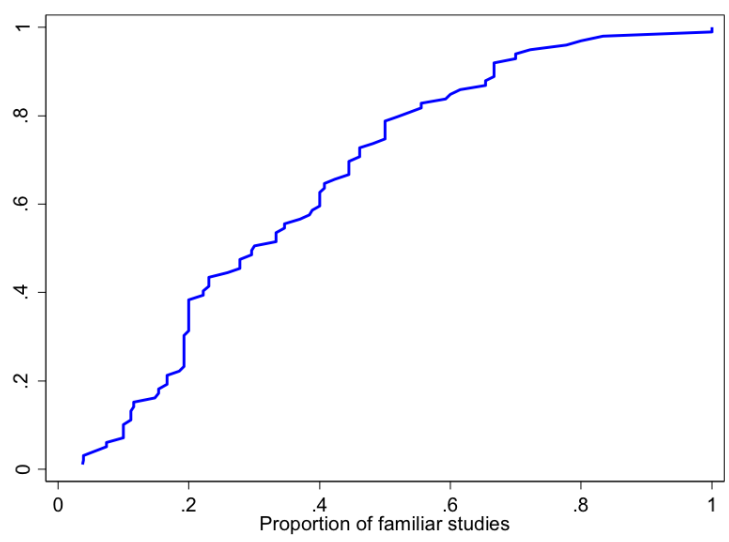

This figure plots the cumulative distribution function for the the proportion of listed studies with which researchers expressed familiarity.

Respondents were only asked to provide estimates of program impact and MDEs for those studies they had not previously heard of. This process of selection meant that lesserknown studies were more likely to be included in our final sample and is the main source of potential bias in the representativeness of our sample set. Table 4 provides a descriptive comparison between studies that were included and excluded in our analysis on this basis. We see that excluded studies tend to be slightly older and slightly less likely to have significant effects. Excluded studies are 3 years older than included studies and approximately 11 percentage points less likely to have a statistically significant result. ${ }^{5}$

\subsection{Predictions and meta-analysis results}

Table 5 summarizes the median of the experts' estimates, minimum detectable effects and meta-analysis results for each intervention-outcome. Expert estimates are larger than meta-analyses results for CCTs, UCTs, deworming and bed nets, but smaller for financial literacy and microfinance. Figure 5 summarizes these findings by plotting the distribution of effect estimates for each intervention-outcome combination relative to the expert estimates

\footnotetext{
${ }^{5}$ We plan to augment this analysis with further observable descriptive statistics in later revisions to the paper.
} 
Figure 4: Example of a list of studies presented to respondents Which of the following subset of studies have you heard the results of (e.g. by reading
the paper/abstract, hearing a presentation, reading a blog post or hearing informally from
a colleague)? Please select all that apply.

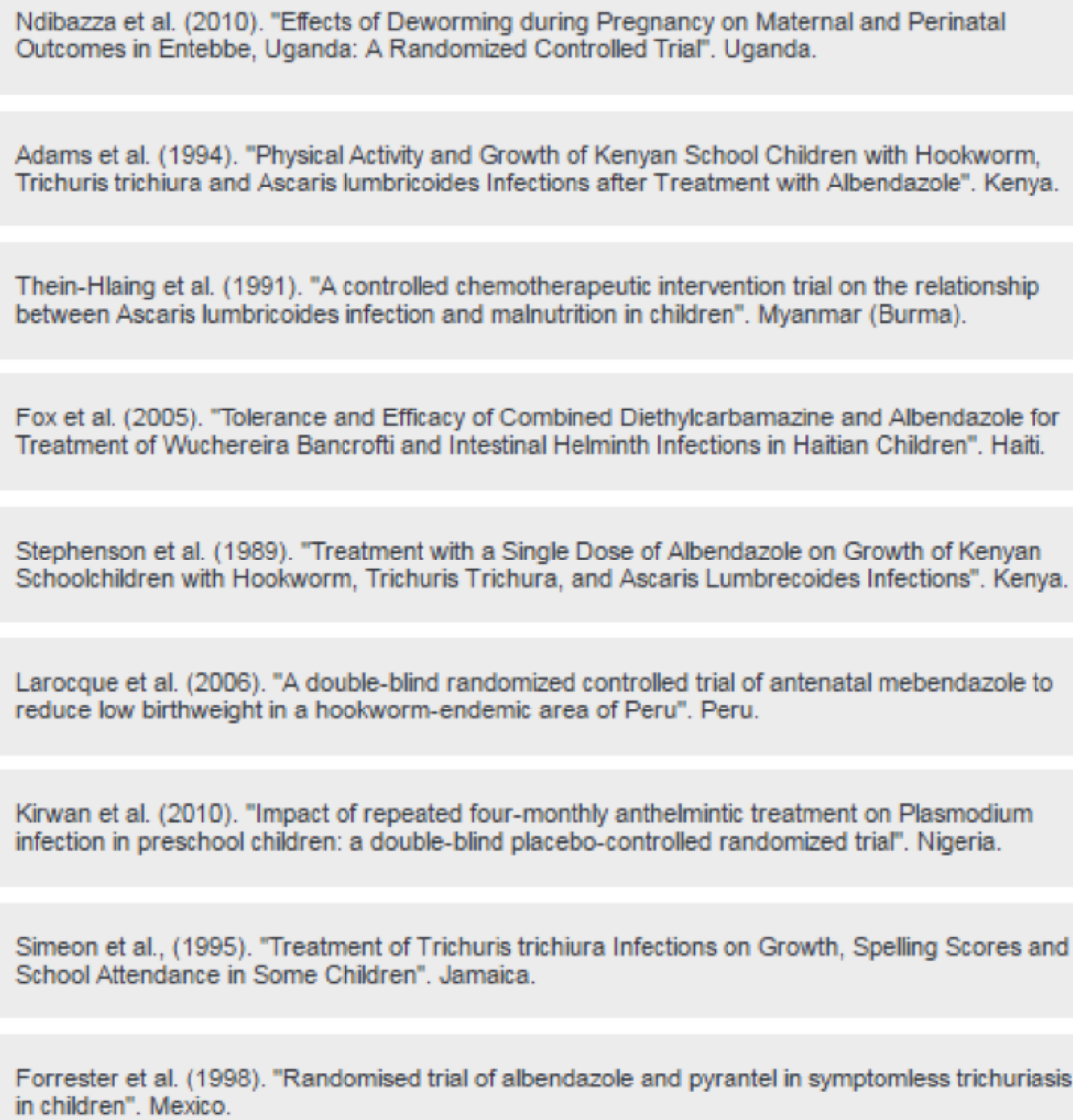

and fixed-effect and random-effects meta-analysis estimates. We find fixed-effect metaanalysis results are systematically lower than random-effects estimates which suggests that larger studies tend to find lower effects, as other literature has documented (e.g. Vivalt, $2017 b)$. 
Table 4: Differences between included and excluded studies

\begin{tabular}{cccc}
\hline & Included & Excluded & Difference in means (p-value) \\
\hline Average publication year & 2007 & 2005 & 0.063 \\
Average p-value & 0.187 & 0.197 & 0.852 \\
Proportion of significant results & $61.9 \%$ & $50.7 \%$ & 0.203 \\
\hline $\mathrm{N}$ & 63 & 67 & - \\
\hline
\end{tabular}

This table compares observable study characteristics between the study results included in the analysis and those excluded because they had too few predictions. The last column presents the p-value of a difference in means test across included and excluded results.

Table 5: Predictions and meta-analysis results by topic

\begin{tabular}{llccccc}
\hline Intervention & Outcome & SR & EE & MDE & FE & RE \\
\hline CCTs & Enrollment (percentage points) & 0.07 & 0.08 & 0.05 & 0.03 & 0.05 \\
UCTs & Enrollment (percentage points) & 0.07 & 0.06 & 0.04 & 0.01 & 0.03 \\
Deworming & Weight (kg) & 0.33 & 0.25 & 0.56 & 0.09 & 0.12 \\
Bed nets & Malaria incidence (odds ratio) & 0.60 & 0.65 & 0.66 & 0.73 & 0.73 \\
Financial literacy & Savings (US\$) & 19.1 & 5.0 & 5.5 & 15.3 & 15.3 \\
Microfinance & Income (US\$) & 23.5 & 25.7 & 40.7 & 22.3 & 24.4 \\
\hline
\end{tabular}

SR is the average impact (study result) found in each intervention-outcome set. EE is the experts' estimated effect, MDE is the minimum detectable effect and FE/RE are the fixed-effect and DerSimonian and Laird random-effects meta-analysis results using all the results in AidGrade's data set. To generate the EE (MDE) we find the average (median) response across respondents for a given study result before calculating intervention-outcome averages, applying equal weights to each study result.

Table 6: Power and reporting errors

\begin{tabular}{lccc}
\hline & EE & FE & RE \\
\hline Power & 0.590 & 0.178 & 0.385 \\
FPRP & 0.001 & 0.008 & 0.004 \\
FNRP & 0.512 & 0.624 & 0.561 \\
Type S error & $<0.001$ & 0.004 & $<0.001$ \\
Type M error & 1.2 & 1.4 & 2.2 \\
\hline
\end{tabular}

This table presents the median power, FPRP, FNRP, Type S and Type M errors across all relevant studies in our sample. This set of statistics is generated using expert estimates of study impact (EE), fixed-effect (FE) and random-effects (RE) meta-analysis. 
Figure 5: Prediction distributions by intervention
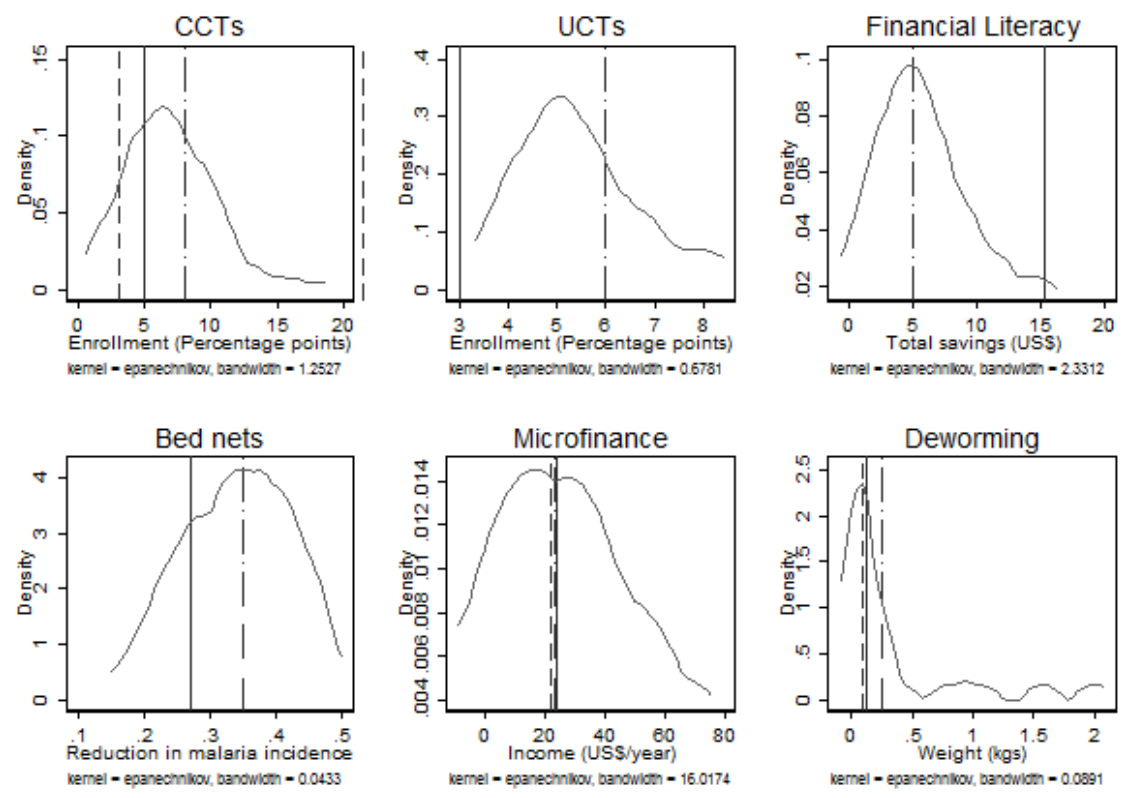

This figure plots the distribution of predictions by intervention and references these distributions against the mean expert estimate (long-dash line), fixed-effect (solid line) and random-effects (short-dash line) meta-analysis results.

\subsection{Power}

We estimate power using two threshold levels: (i) the average predicted effect and (ii) the respective random-effects and fixed-effect meta-analysis results. Figure 6 presents the power cumulative distribution across the sample. The median power to detect an average predicted effect is 0.59 with $33 \%$ having power greater than 0.8 . Using meta-analysis results as the threshold effect yields median power estimates between 0.178 (fixed-effect) and 0.385 (random-effects). Our results using a fixed-effect threshold are comparable to Ioannidis et al. (forthcoming). However, the expert prediction and random-effects approaches generate significantly larger power estimates. If we believe the expert predictions or meta-analysis results overestimate the true underlying program impacts, it is possible we may be overestimating power to detect true effects. Regardless, these calculations are substantially lower 
than a power of 0.8 that is typically seen as a benchmark for empirical research. We note, however, that studies may not be specifically interested in the outcomes we are exploring and we should be careful not to pass normative judgment on these numbers.

Figure 6: Distribution of power

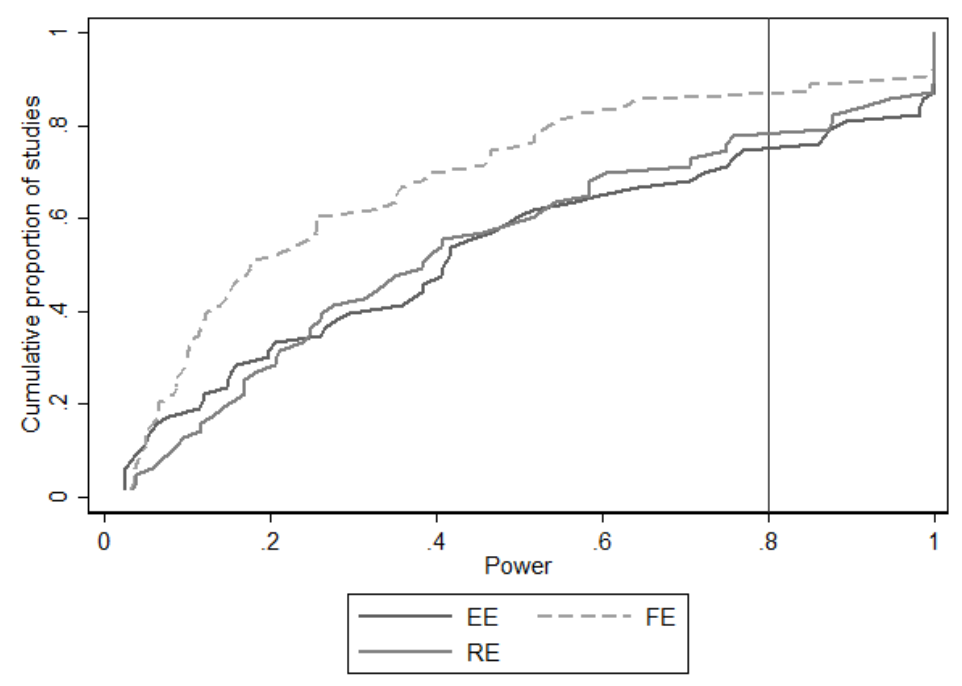

This figure plots the cumulative distribution of study power using 3 threshold levels: (i) the average predicted estimated impact; (ii) the fixed-effect meta-analysis estimate; and (iii) the random-effect meta-analysis estimate. A vertical line indicating power $=0.8$ is included for reference.

\subsection{FPRP and FNRP estimates}

Using Equation 1, we estimate the FPRP for all study results with p-values less than or equal to 0.05 ( $62 \%$ of the results) and similarly calculate the FNRP for study results with p-values higher than 0.05 .

The median FPRP for this set of papers using expert predictions is 0.001 - the same as the median p-value for significant results in our sample; the median FNRP is 0.512. The FPRP error rate is substantially smaller than estimates for other disciplines where similar exercises has been done.

Using meta-analysis results rather than expected effects for the power calculations, these numbers remain relatively stable: the median FPRP ranges from 0.004 (random-effects) to 
0.008 (fixed-effect meta-analysis), while the median FNRP ranges from 0.561 (randomeffects) to 0.624 (fixed-effect).

\subsection{Type $\mathrm{S}$ and Type $\mathrm{M}$ errors}

The median value for Type $\mathrm{S}$ errors using expert predictions is $<0.001-i . e$. it is very unlikely that a significant result would have the wrong sign. The median value using the random-effects meta-analysis result for the prior was 0.0002 , and the median value using the fixed-effect meta-analysis result was 0.005 .

The median value for the Type $M$ exaggeration factor using expert predictions to form the prior is 1.2 ; using the random-effects meta-analysis result for the prior, this is 2.2 ; using the fixed-effect meta-analysis result, 1.4. This indicates that while the sign of a significant result is likely correct, point estimates may often be inflated.

\subsection{Seminar series results}

We find a median power for the 8 study results of 0.72 . Of the 8 results, 5 were significant. The median FPRP is 0.016 and the FNRP is 0.423 . The median probability of a Type $\mathrm{S}$ error for significant results is negligible and the median Type M exaggeration factor is 1.1.

Clearly, this sample is too small to make any inferential claims, but the results are aligned with message coming out of the findings from the expert prediction exercise - namely, that positive results appear credible from a statistical perspective. The lay respondents in this exercise provided optimistic estimates on average. The median prediction was larger than the actual impact evaluation result in 6 of the 8 cases and the probability that an effect larger than the MDE will be found was anticipated to be relatively high (0.46). Meta-analysis results that could provide additional benchmarks do not exist for these studies. 


\section{Discussion}

While power remains problematic, this does not substantial effect false positive rates, and the estimates are better than anticipated. However, we may think that our results represent an especially optimistic view. In particular, we again note that the majority of the studies we ultimately considered were CCTs or UCTs, and these had very large sample sizes and correspondingly low p-values. We can imagine other sub-disciplines within development economics may yield less promising results because of the sample size and take up challenges associated with them. For instance, Mckenzie and Woodruff (2013) identify small sample sizes in business training programs for microenterprises to be an inhibiting factor in the credibility of research findings on the topic.

Further, the overall sample is only representative of development economics interventions that have already been subjected to a critical mass of impact evaluations. We may worry that by virtue of selecting those intervention-outcome combinations covered by the greatest number of papers, we are selecting outcomes that may a priori be more likely to be affected by the intervention. Estimates of the FPRP would then represent a lower bound. Still, we find it worthwhile to consider the most commonly-reported outcomes, precisely because they are the most common and likely of primary interest. A further selection challenge is that well-known papers are likely to be under-represented since we are only able to collect priors on studies with which people are not familiar. The direction of this bias is ambiguous. If studies are better-known because of the overall quality of the design (e.g. large sample sizes), then we may be overestimating reporting error. If, alternatively, papers are well-known because of surprising findings (low priors relative to the estimated effects) then we may be underestimating reporting error. Nonetheless, we did not observe any significant differences between those papers that were known and those that were unknown, except perhaps for a suggestion of a recency bias $(\mathrm{p}<0.10)$.

A second, separate issue is that we may believe that expert priors systematically overestimate true effects. In this case, we would underestimate the extent of false reports by 
artificially inflating power when using these predictions to define the threshold. To mitigate this concern, we presented results using meta-analysis results for comparison. These too may be inflated if we think that publication bias makes meta-analysis results overoptimistic.

Finally, much of this paper relies on data collected after studies were completed and we are unable to assess how much people may have been indirectly influenced by study findings even if they did not have conscious knowledge of the study. Clearly, it would be better to use a large set of priors and power calculations collected ex ante for a broad array of studies. DIME is systematically collecting priors for studies in its portfolio going forward, and future work could leverage these data. We hope that at some point in the future, another organization can systematically gather this information on a broader scale.

Collecting priors can be beneficial to policy-focused empirical research in a number of ways beyond ex post assessments of research credibility. First, collecting priors can help research teams more realistically assess the potential power of a study ex ante and provide an important signal for whether a research design may need to be revised. Second, assuming the studies are adequately powered, having priors provides a structured way for showing how a new piece of research either supports or contradicts original beliefs. This can be a valuable tool for motivating the contribution of a paper, particularly for null results that may not have been anticipated, or results that seem obvious ex post but may not have been so ex ante. Finally, the collection of priors provides researchers with an entry point to apply Bayesian approaches to estimate the effects of their program - a practice currently all but non-existent in the development economics literature, but primed for growth.

\section{Conclusion}

One of the most frequently-reported aspects of a study is whether or not its results were statistically significant. We estimate a series of statistics on false reporting in development economics to emphasize the importance of looking beyond p-values when assessing the 
credibility of a result. In particular, we highlight the value of incorporating priors on intervention impacts and considering study power to detect effects of these magnitudes to determine the extent of potential reporting errors. We find that the majority of studies reviewed fair exceptionally well, particularly when referenced against other disciplines that have performed similar exercises.

While this paper estimates the likelihood of false reporting in development economics, the results are only illustrative, and other studies in development economics may still prove to be less credible. The broader contribution is to highlight how analysis of study power and the systematic collection of priors can help assess the quality of research. 


\section{References}

Begley, C.G. and Ioannidis, J.P., 2015. "Reproducibility in science." Circulation research, 116(1), pp.116-126.

Brodeur, A., L, M., Sangnier, M. and Zylberberg, Y., 2016. "Star wars: The empirics strike back." American Economic Journal: Applied Economics, 8(1), pp.1-32.

Button, K. S., Ioannidis, J. P. A., Mokrysz, C., Nosek, B. A., Flint, J., Robinson, E. S., \& Munaf, M. R. 2013. "Power failure: why small sample size undermines the reliability of neuroscience." Nature Reviews Neuroscience, 14(5), 365-376.

Camerer, C.F., Dreber, A., Forsell, E., Ho, T.H., Huber, J., Johannesson, M., Kirchler, M., Almenberg, J., Altmejd, A., Chan, T. and Heikensten, E., 2016. "Evaluating replicability of laboratory experiments in economics." Science, 351(6280), pp.1433-1436.

De Andrade, G.H., Bruhn, M. and McKenzie, D., 2014. "A helping hand or the long arm of the law? Experimental evidence on what governments can do to formalize firms." The World Bank Economic Review, p.lhu008.

DellaVigna, S. and Pope, D., 2016a. "What motivates effort? Evidence and expert forecasts" (No. w22193). National Bureau of Economic Research.

DellaVigna, S. and Pope, D., 2016b. "Predicting Experimental Results: Who Knows What?" (No. w22566). National Bureau of Economic Research.

DerSimonian, R. and Laird, N., 1986. "Meta-analysis in clinical trials." Controlled clinical trials, $7(3)$, pp.177-188.

Dreber, A., Pfeiffer, T., Almenberg, J., Isaksson, S., Wilson, B., Chen, Y., Nosek, B.A. and Johannesson, M., 2015. "Using prediction markets to estimate the reproducibility of scientific research." Proceedings of the National Academy of Sciences, 112(50), pp.15343-15347. Groh, M., Krishnan, N., McKenzie, D. and Vishwanath, T., 2016. "The impact of soft skills training on female youth employment: evidence from a randomized experiment in Jordan." IZA Journal of Labor Development, 5(1), pp.9.

Gelman, A. and Carlin, J., 2014. "Beyond power calculations assessing type S (sign) and 
type M (magnitude) errors." Perspectives on Psychological Science, 9(6), pp.641-651.

Gelman, A. and Tuerlinckx, F., 2000. "Type S error rates for classical and Bayesian single and multiple comparison procedures." Computational Statistics, 15, pp.373-390. doi:10.1007/s001800000040.

Hirshleifer, S., McKenzie, D., Almeida, R. and RidaoCano, C., 2015. "The impact of vocational training for the unemployed: experimental evidence from Turkey." The Economic Journal.

Hoenig, J.M. and Heisey, D.M., 2001. "The abuse of power." The American Statistician, $55(1), 19-24$.

Ioannidis, J.P., 2008. "Why most discovered true associations are inflated." Epidemiology, 19(5), pp.640-648.

Ioannidis, J. P. A., Stanley, T. D., Hristos, D. (forthcoming). "The power of bias in economics research." Economic Journal.

Ioannidis, J. P. A. 2005. "Why Most Published Research Findings Are False." PLoS Med 2 (8): e124. doi:10.1371/journal.pmed.0020124.

McKenzie, D. and Woodruff, C., 2013. "What are we learning from business training and entrepreneurship evaluations around the developing world?." The World Bank Research Observer, 29(1), pp.48-82.

McKenzie, D. (forthcoming). Can business owners form accurate counterfactuals? eliciting treatment and control beliefs about their outcomes in the alternative treatment status. Journal of Business Economic Statistics.

Open Science Collaboration, 2015. "Estimating the reproducibility of psychological science." Science, 349(6251), p.aac4716.

Rosenthal, R., 1979. "The file drawer problem and tolerance for null results." Psychological bulletin, 86(3), p.638.

Schulz, K.F. and Grimes, D.A., 2005. "Sample size calculations in randomised trials: mandatory and mystical." The Lancet, 365(9467), pp.1348-1353. Smaldino, P.E. and McElreath, R., 2016. "The natural selection of bad science." Royal Society Open Science, 3(9), 
p.160384.

Vivalt, E. 2017a. "The Trajectory of Specification Searching and Publication Bias Across Methods and Disciplines." Unpublished manuscript.

Vivalt, E. 2017b. "How Much Can We Generalize from Impact Evaluations?" Unpublished manuscript.

Wacholder, S., Chanock, S., Garcia-Closas, M., \& Rothman, N. 2004. "Assessing the probability that a positive report is false: an approach for molecular epidemiology studies." Journal of the National Cancer Institute, 96(6), 434-442. 


\section{Appendix 1: Future Extensions}

There is a trade-off between the validity of priors collected and the comprehensiveness of the studies included in the analysis to reflect the stock of literature. The current paper presents the results from expert predictions on impact evaluations that have already been completed, but of which the expert is unaware. This has the benefit of leveraging a large set of studies, but brings with it a concern of potential bias. Respondents may not perfectly recall which studies they have heard about. In that case, their measured "priors" could actually reflect their posteriors. Second, since the current approach focuses on experts in their respective fields, we may only receive responses to more obscure (and potentially less influential) studies since we require that they have not heard of them, limiting the potential representativeness of the results.

Attempting to gather the required information from design, pre-analysis plans and concept note documents created before results were generated was not feasible upon surveying what ex ante evidence was available. Most design notes do not include explicit predictions of intervention effect sizes. Where ex ante power calculations have been done, they generally used a MDE as the threshold value for the calculation, rather than the estimate of the true program effect. We are concerned that researchers may have adjusted their expectations based on the sample size constraints and other operational realities, rather that on their true beliefs, in what Schultz \& Grimes (2005) describe as the "sample size samba".

Thus, we would prefer to ideally gather priors for ongoing interventions before the results are produced. Towards this objective, we plan to collect responses from policymakers, practitioners and researchers for ongoing impact evaluations that have not yet collected data or have not yet made results public.

For this set of projects, we will ask the researchers and World Bank operational staff, as well as the government counterparts involved in each project, to provide their priors, confidence in their priors, and MDE for the project they are working on. DIME plans to gather this information systematically as part of the monitoring of their portfolio of impact 
evaluations. The first round of data collection is anticipated for the summer of 2017, where all projects that have completed at least a baseline will be asked for this information. We anticipate that this will cover approximately 50 projects. In the longer term, we aim to compare these priors to actual results generated from the respective studies. 


\section{Appendix 2: Additional Tables and Figures}

Table A1: List of Interventions and Outcomes Covered

\begin{tabular}{ll}
\hline Intervention & Outcome \\
\hline Conditional cash transfers & School enrollment rates \\
Deworming & Weight \\
Financial literacy & Savings \\
Insecticide-treated bed nets & Malaria \\
Microfinance & Income \\
Unconditional cash transfers & School enrollment rates \\
Water treatment & Diarrhea prevalence \\
\hline
\end{tabular}

Table A2: Power and reporting errors based on pre-analysis specifications

\begin{tabular}{lc}
\hline & Expert estimate \\
\hline Power & 0.382 \\
FPRP & 0.018 \\
FNRP & 0.265 \\
\hline
\end{tabular}

This table presents the results based on the specifications described in the pre-analysis plan. We calculate power using the median minimum detectable effect identified for each topic. We calculate the FPRP and FNRP by defining the prior as the proportion of predictions lying above the median minimum detectable effect. 


\section{Appendix 3: Departures from Pre-Analysis Plan}

First, we originally anticipated to collect priors through 3 different approaches including: (i) survey responses on completed studies from experts; (ii) an email poll sent to all participants in the World Bank's Development Impact Evaluation (DIME) group mailing list about the impact of different impact evaluations presented at DIME lunchtime seminars; and (iii) feedback from operational, policy and research counterparts involved in 10 impact evaluations at the World Bank that are yet to be completed. This paper presents the results from (i) and (ii), while (iii) is ongoing and will be presented when the data become available.

The second deviation from the original design was to collect priors on the link between micronutrients and anemia. Micronutrients was excluded as a topic since it has been studied mostly in the context of evaluations in public health and had such a large number of studies that we feared including it would shift the focus of the paper from economics to health.

Third, we initially anticipated using the minimum detectable effect as the threshold for which power would be calculated. This was changed to using the meta-analysis results and expert predictions advice for ex post power calculations described in Gelman \& Carlin (2014).

Finally, we revised our original approach to deriving the prior distribution used to assess priors for the FPRP and FNRP due to sample size limitations. While we originally proposed calculating the prior probability of the intervention having a significant impact by observing the number of expert estimates above the MDE threshold, we realized that this would create many instances where priors would be estimated to be one or zero (where the MDE lay outside the range of prior estimates). To avoid the loss of data we assign values of 0.01 to priors where none of the predictions are larger than the median MDE, and a value of 0.99 if all of the predictions are larger than the MDE.

Since the third and fourth deviations from the pre-analysis plan are statistical, we re-run the analysis using the originally specified approaches and present these in an appendix for 
reference. 